\title{
Article \\ Acyl Chain Specificity of Marine Streptomyces klenkii PhosPholipase D and Its Application in Enzymatic Preparation of Phosphatidylserine
}

\author{
Rongkang Hu, Ruiguo Cui, Dongming Lan, Fanghua Wang * and Yonghua Wang *
}

Citation: Hu, R.; Cui, R.; Lan, D.; Wang, F.; Wang, Y. Acyl Chain Specificity of Marine Streptomyces klenkii PhosPholipase D and Its Application in Enzymatic Preparation of Phosphatidylserine. Int. J. Mol. Sci. 2021, 22, 10580. https://doi.org/ 10.3390/ijms221910580

Received: 19 August 2021

Accepted: 18 September 2021

Published: 30 September 2021

Publisher's Note: MDPI stays neutral with regard to jurisdictional claims in published maps and institutional affiliations.

Copyright: (C) 2021 by the authors. Licensee MDPI, Basel, Switzerland. This article is an open access article distributed under the terms and conditions of the Creative Commons Attribution (CC BY) license (https:// creativecommons.org/licenses/by/ $4.0 /)$.
School of Food Science and Engineering, South China University of Technology, Guangzhou 510640, China; 201810106652@mail.scut.edu.cn (R.H.); 201810106665@mail.scut.edu.cn (R.C.); dmlan@scut.edu.cn (D.L.)

* Correspondence: wangfanghua@scut.edu.cn (F.W.); yonghw@scut.edu.cn (Y.W.); Tel.: +86-20-8711-3848 (F.W.); +86-20-8711-3842 (Y.W.)

\begin{abstract}
Mining of phospholipase D (PLD) with altered acyl group recognition except its head group specificity is also useful in terms of specific acyl size phospholipid production and as diagnostic reagents for quantifying specific phospholipid species. Microbial PLDs from Actinomycetes, especially Streptomyces, best fit this process requirements. In the present studies, a new PLD from marine Streptomyces klenkii (SkPLD) was purified and biochemically characterized. The optimal reaction temperature and $\mathrm{pH}$ of SkPLD were determined to be $60^{\circ} \mathrm{C}$ and 8.0, respectively. Kinetic analysis showed that SkPLD had the relatively high catalytic efficiency toward phosphatidylcholines (PCs) with medium acyl chain length, especially 12:0/12:0-PC $\left(67.13 \mathrm{~S}^{-1} \mathrm{mM}^{-1}\right)$, but lower catalytic efficiency toward PCs with long acyl chain ( $>16$ fatty acids). Molecular docking results indicated that the different catalytic efficiency was related to the increased steric hindrance of long acyl-chains in the substrate-binding pockets and differences in hydrogen-bond interactions between the acyl chains and substrate-binding pockets. The enzyme displayed suitable transphosphatidylation activity and the reaction process showed $26.18 \%$ yield with L-serine and soybean PC as substrates. Present study not only enriched the PLD enzyme library but also provide guidance for the further mining of PLDs with special phospholipids recognition properties.
\end{abstract}

Keywords: phospholipase D; enzyme characterization; chain length specificity; molecular docking; phosphatidylserine

\section{Introduction}

Phospholipids (PLs) are one kinds of mixed lipids containing phosphoric acid, which are the basic component of cell membrane and the essential substance of life [1]. PLs have been broadly applied in emulsifiers, components of cosmetics, medical formulations and for liposome preparations because of their unique chemical structure and healthcare functions [2]. In recent years, due to the increasing cost of healthcare-related products and expanding health benefits of functional lipids, there is increasing interest in PLs with varying molecular properties (such as charge, polarity, size, etc.) to obtain special functional PLs with excellent process properties and outstanding physiological and pharmacological functions [3]. Enzymatic modification of natural PLs has become an important way to realize the high value utilization of PLs.

PLs consist of a glycerol backbone and a polar head group located at the sn-3 position of the glycerol backbone. Phospholipase D (PLD, EC 3.1.4.4) hydrolyze the phosphodiester bond between phosphate at the $s n-3$ position of PL and the polar head groups. PLDcatalyzed transphosphatidylation is especially useful in synthesis of naturally less abundant PLs such as phosphatidylglycerol (PG), phosphatidylethanolamine (PE), phosphatidylserine (PS), and phosphatidylinositol (PI) by exchanging choline head group of phosphatidylcholine (PC) [4-7]. In addition, PLD with altered acyl group recognition except its head 
group specificity is also useful for the production of specific PL products and as diagnostic reagents for quantifying specific PL species [8]. PLs can be classified as short, medium and long acyl chains based on the fatty acid chain lengths at $s n-1$ and $s n-2$ positions of the glycerol backbone. The molecular properties and biological reactivity of acyl chain are strongly influenced by the length of fatty acid chain [9]. Strong interested in understanding the mechanism of acyl group recognition by the enzyme, and further alteration of the acyl group specificity are rising. PLDs from Streptomyces species (PLDStr) are mostly used for this purpose due to their higher transphosphatidylation activity and broader substrate specificity compared to other microbial PLDs $[10,11]$. According to the NCBI database (https: / / www.ncbi.nlm.nih.gov / protein/?term=Streptomyces+Phospholipase+D) (accessed on 16 September 2021), currently there are 8099 protein sequences from Streptomyces in the PLD superfamily. However, until now, only about 20 PLDs from Streptomyces were experimentally characterized. Streptomyces are found in a wide range of aquatic and terrestrial environments, it have the metabolic pathways with the abundant hydrolytic enzymes [12,13]. However, most of the reported PLDs originated from Streptomyces live in terrestrial soil environments. Recently, the conventional screening and metagenome strategies have been developed to discover potential Streptomyces PLDs. Zhou et al. established 3 screening criteria for amino acid sequence identity, conserved motifs and availability of organism base on genome sequencing and annotation in the database. Based on 3 screening criteria, a new PLD was successfully mined from Streptomyces mobaraensis, which can catalyze soybean lecithin and L-serine into PS [14]. Although some achievements have been made, compared with a large amount of sequence information in this family, the extreme lack of enzymatic properties information serious hindered the in-depth understanding of the structure-function relationship and further application of this enzyme.

In the present study, a new PLD from marine Streptomyces klenkii (SkPLD) was biochemical characterized. With the help of molecular docking, the molecular basis for acyl chain specificity of SkPLD was illustrated. Meanwhile, the application potential of SkPLD for the synthesis of PS was evaluated. Present investigations not only further enriched the enzyme library but also provide important information on the understanding of the structure-function relationship of SkPLD.

\section{Results}

\subsection{Bioinformatic Analysis of SkPLD}

The full-length SkPLD contains 539 amino acids and the first 33 amino acids were predicted to be the signal peptide (Figure S1), indicating that the enzyme was an extracellular protein. All known Streptomyces PLDs, except the S. chromofuscus PLD, contain two strictly conserved HKD motifs $\left(\mathrm{HXK}(\mathrm{X})_{4} \mathrm{D}(\mathrm{X})_{6} \mathrm{GG} / \mathrm{S}\right)$, which are assumed to act as active center [15]. SkPLD showed substantially high identity to PLD protein sequences from Streptomyces (>70\%) (Figures 1 and S2). SkPLD also contained the typical active site of the PLD superfamily. Three catalytic residues (histidine, lysine and aspartic acid) of SkPLD formed the conserved sequence motif $\mathrm{HXK}(\mathrm{X})_{4} \mathrm{D}$ by the multiple alignment analysis.

\subsection{Expression and Purification of SkPLD}

The mature SkPLD (without signal peptides) was heterologously expressed in E. coli Shuffle $\mathrm{T} 7$ and then purified to homogeneity by using $\mathrm{Ni}^{2+}$ affinity chromatography, anion exchange chromatography and gel filtration chromatography (Figure S3). SDS-PAGE analysis revealed that recombinant SkPLD was approximately $54 \mathrm{kDa}$, which matched the theoretical molecular weight well. The protein contents and catalytic activities during the purification process were documented in Table S1. About 1.24 mg of pure SkPLD was obtained from $100 \mathrm{~g}$ wet cell weight, and the specific activity of pure SkPLD using soybean $\mathrm{PC}$ as substrate was determined to be $26.93 \mu \mathrm{mol} / \mathrm{min} / \mathrm{mg}$. 


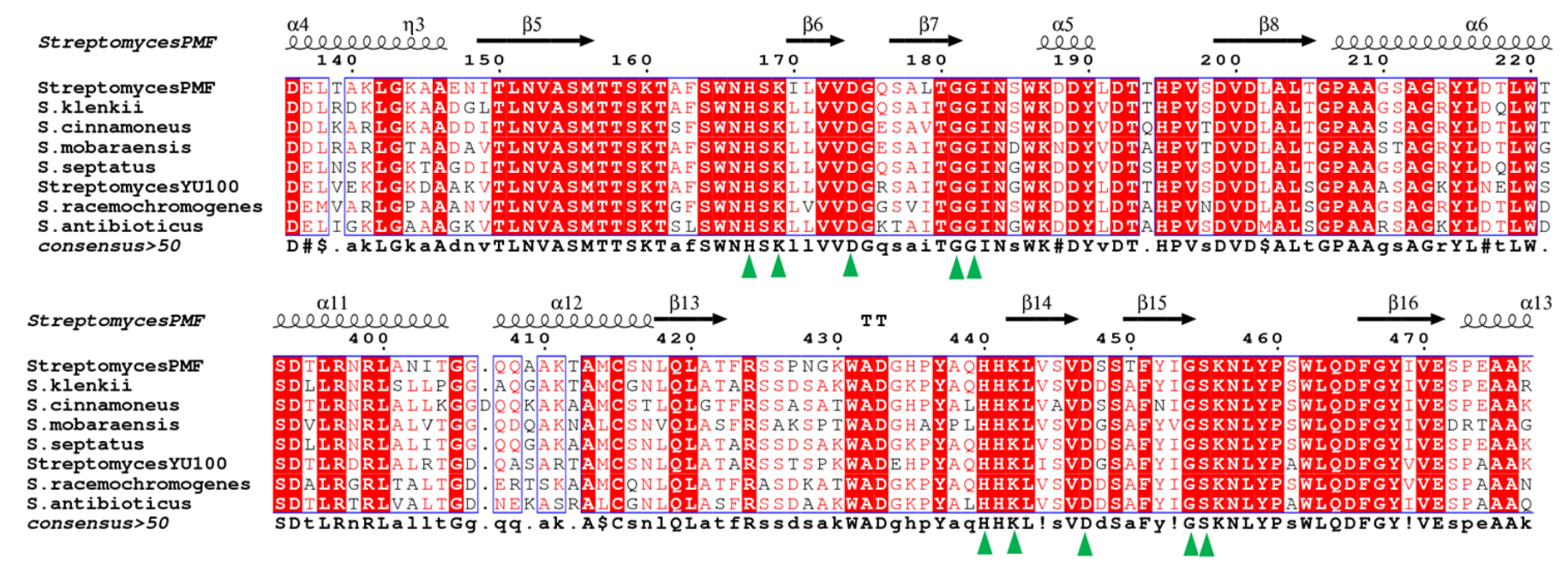

Figure 1. Multiple amino acid sequence alignment of SkPLD conserved region with several other Streptomyces PLDs. Secondary structures of PLDs were shown above the alignments. Residues highlighted in red background were indentical among the protein compared. Residues in the conserved HxKxxxxDxxxxxxGG/S (HKD) motifs of the PLD superfamily were indicated with green triangles. Arrow stands for $\beta$-strand, helical curve denotes $\alpha$-helix.

\subsection{Enzymatic Characterization of SkPLD}

SkPLD was found to be an alkaline PLD, with a maximal activity at $\mathrm{pH} 8.0$, and exhibited lower (about $40 \%$ of the maximum activity) at $\mathrm{pH} 7.0$ and $\mathrm{pH} 10.0$ (Figure 2A). SkPLD showed wide pH stability between $7.0-10.0$, with more than $75 \%$ of residual activities left after $2 \mathrm{~h}$ treatment under corresponding $\mathrm{pH}$ buffers (Figure 2B). As shown in Figure 2C, the maximum hydrolytic activity of SkPLD was found at $60^{\circ} \mathrm{C}$, whereas its activity decreased to $40 \%$ of the maximum under the temperature of $70{ }^{\circ} \mathrm{C}$. SkPLD kept stable after incubation at temperatures lower than $35^{\circ} \mathrm{C}$ for $4 \mathrm{~h}$, with a residual activity higher than $80 \%$ (Figure 2D). SkPLD kept more than $84.75 \%$ of the initial activity while the existence of different metal ions $(10 \mathrm{mM})$ in the buffer, including $\mathrm{Na}^{+}, \mathrm{K}^{+}, \mathrm{Cu}^{+}, \mathrm{Li}^{+}$and $\mathrm{Zn}^{2+}$. As metal ions concentration increases from $1 \mathrm{mM}$ to $5 \mathrm{mM}$, the hydrolytic activity of SkPLD was activated in the presence of $\mathrm{Co}^{2+}, \mathrm{Ca}^{2+}$ and $\mathrm{Mn}^{2+}$ but was inhibited by $\mathrm{Al}^{3+}$ and $\mathrm{Fe}^{2+}$. The enzyme activity of SkPLD kept $85.66 \%$ even in the existence of $10 \mathrm{mM}$ EDTA (Table S2).

\subsection{Kinetic Parameters and Acyl Chain Specificity of SkPLD}

The kinetic parameters of SkPLD were determined with soybean PC or PCs with different chain lengths as substrate. The enzyme showed hyperbolic $v /[S]$-curves, which could be fitted well according to the classical Michaelis-Menten function by non-linear regression (Figure S4). The apparent $V_{\max }$ and $K_{m}$ values were determined to be $41.65 \pm 1.84 \mu \mathrm{mol} / \mathrm{min} / \mathrm{mg}$ and $1.33 \pm 0.13 \mathrm{mM}$, respectively. The influence of the acyl chain length in PC on the kinetic parameters of SkPLD was also investigated (Table 1). The enzymes exhibited the highest catalytic efficiency $\left(k_{\mathrm{cat}} / K_{\mathrm{m}}\right)$ toward PCs with medium acyl chain followed by short and long acyl chain. SkPLD showed a maximum catalytic efficiency $\left(k_{\text {cat }} / K_{\mathrm{m}}\right)$ toward the 12:0/12:0-PC $\left(67.13 \mathrm{~S}^{-1} \mathrm{mM}^{-1}\right)$ and $k_{\text {cat }} / K_{\mathrm{m}}$ value decreased sharply as the substrate changed from 12:0/12:0-PC to 18:0/18:0-PC. 
A

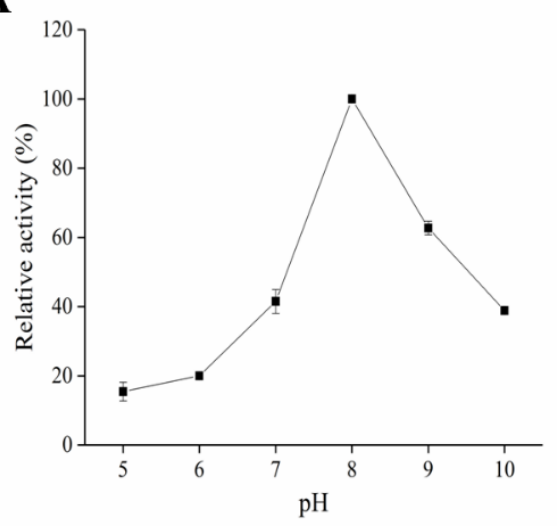

C

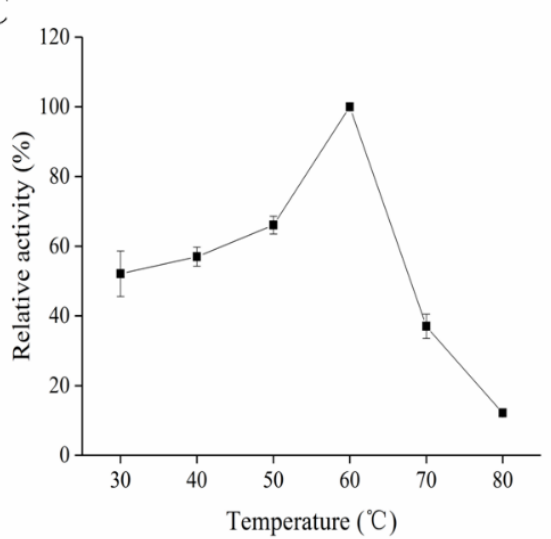

B

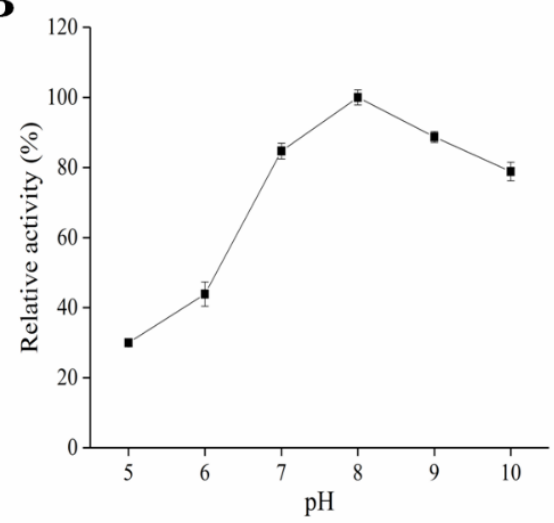

D

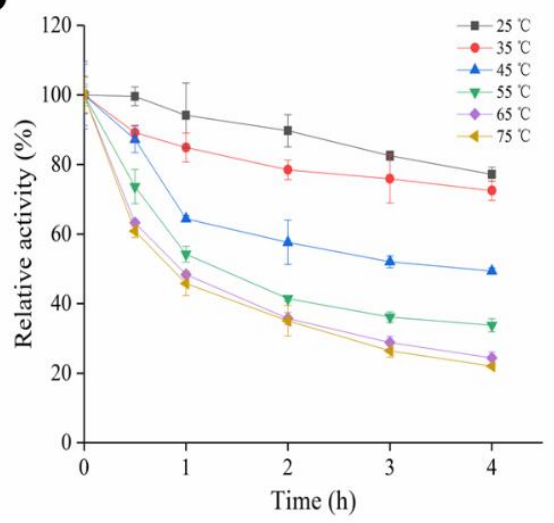

Figure 2. Effects of $\mathrm{pH}$ and temperature on the hydrolytic activity of SkPLD. (A) The enzymatic activity of SkPLD was measured under various $\mathrm{pH}$ buffers. The enzymatic activity value obtained at pH 8.0 was taken as $100 \%$. (B) pH stability of SkPLD. The residual activity of SkPLD was determined after treating for $2 \mathrm{~h}$ under various $\mathrm{pH}$ buffers. The residual activity value obtained at $\mathrm{pH} 8.0$ was taken as $100 \%$. (C) The enzymatic activity of SkPLD was measured at various temperatures. The enzymatic activity value obtained at $60^{\circ} \mathrm{C}$ was taken as $100 \%$. (D) Thermostability of SkPLD. The residual activity was determined after treating for $4 \mathrm{~h}$ at different temperatures. Values were means \pm standard deviation from three independent experiments.

Table 1. Kinetic parameters for the hydrolysis of SkPLD towards different substrates.

\begin{tabular}{cccc}
\hline Substrates & $\boldsymbol{K}_{\mathbf{m}}(\mathbf{m M})^{\mathbf{a}}$ & $\boldsymbol{k}_{\text {cat }}\left(\mathbf{S}^{-\mathbf{1}} \mathbf{b}^{\mathbf{b}}\right.$ & $\boldsymbol{k}_{\text {cat }} / \boldsymbol{K}_{\mathbf{m}}\left(\mathbf{S}^{-\mathbf{1}} \mathbf{m} \mathbf{M}^{-\mathbf{1}}\right)^{\mathbf{c}}$ \\
\hline 6:0/6:0-PC & $2.01 \pm 0.18$ & $100.85 \pm 4.42$ & 50.27 \\
8:0/8:0-PC & $2.59 \pm 0.28$ & $110.54 \pm 6.30$ & 42.63 \\
12:0/12:0-PC & $1.09 \pm 0.10$ & $73.29 \pm 2.68$ & 67.13 \\
14:0/14:0-PC & $1.07 \pm 0.18$ & $61.65 \pm 4.05$ & 57.51 \\
16:0/16:0-PC & $2.01 \pm 0.19$ & $32.97 \pm 1.48$ & 16.43 \\
18:0/18:0-PC & $1.27 \pm 0.14$ & $15.45 \pm 0.69$ & 12.12 \\
\hline
\end{tabular}

${ }^{\mathrm{a}} K_{\mathrm{m}}$ : the substrate affinity constant. ${ }^{\mathrm{b}} k_{\mathrm{cat}}$ : the turnover of the enzymatic reaction. ${ }^{\mathrm{c}} k_{\mathrm{cat}} / K_{\mathrm{m}}$ : the catalytic efficiency.

\subsection{Structural Feature and Molecular Basis for Chain Length Specificity}

The structural quality of the model for SkPLD was validated to ensure that it was suitable for performing further studies. As shown in Figure S5A, the overall quality of the model was also evaluated by the score obtained from QMEAN server. QMEAN4 score, which is a composite score consisting of a linear combination of 4 statistical potential terms. This value falls in the range of estimated model reliability score between 0 and 1 . The normalized QMEAN4 Z-score for the model was found to be 0.87 . Thus, the quality 
evaluation using online tools QMEAN confirmed a proper model conformation and of good quality for further analysis. As shown in Figure S5B, SkPLD contained the catalytic quadruplex amino acid residues His167, Lys169, Asn184, His440, Lys442 and Asn457. They were in similar locations to those of the crystal structure of PLD from S. antibioticus (PDB ID: 2ZE4) and Streptomyces sp. PMF (PDBID: 1F0I), demonstrating a proper model conformation. The docking study of the substrate PCs against PLD was performed, and the results are displayed in Figure 3 and Table 2. The hydrogen-bond interactions between SkPLD and PCs was initially increased with increasing length of acyl chain from 6:0/6:0-PC to 12:0/12:0-PC and then decreased with increasing length of acyl chain from 12:0/12:0-PC to 16:0/16:0-PC. The absolute value of estimated binding energy increased from 7.10 to $7.54 \mathrm{kcal} / \mathrm{mol}$ and then decreased from 7.54 to $6.79 \mathrm{kcal} / \mathrm{mol}$ for the SkPLD-PCs complex as increasing chain length from 6:0/6:0-PC to 16:0/16:0-PC. SkPLD and 16:0/16:0-PC complex showed four hydrogen bonds. However, SkPLD and 12:0/12:0-PC complex had eight hydrogen bond with an estimated binding energy of $-7.54 \mathrm{kcal} / \mathrm{mol}$, which is the highest binding affinity. SkPLD and 18:0/18:0-PC complex obtained binding energy of $-7.28 \mathrm{kcal} / \mathrm{mol}$ and relatively high hydrogen-bond interaction, which were consistent with its substrate affinity in Table 1.
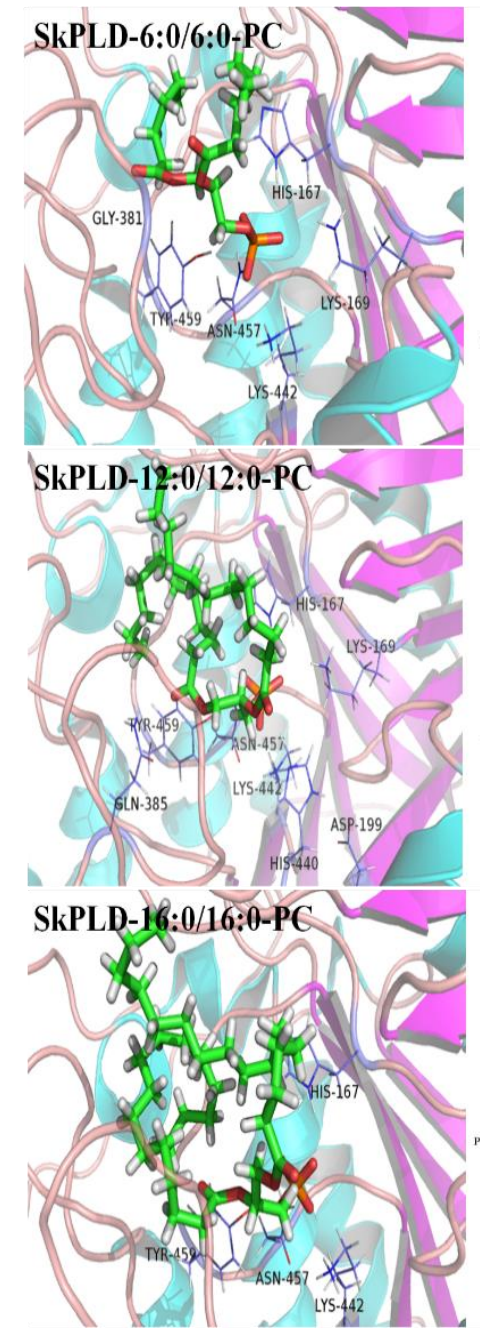
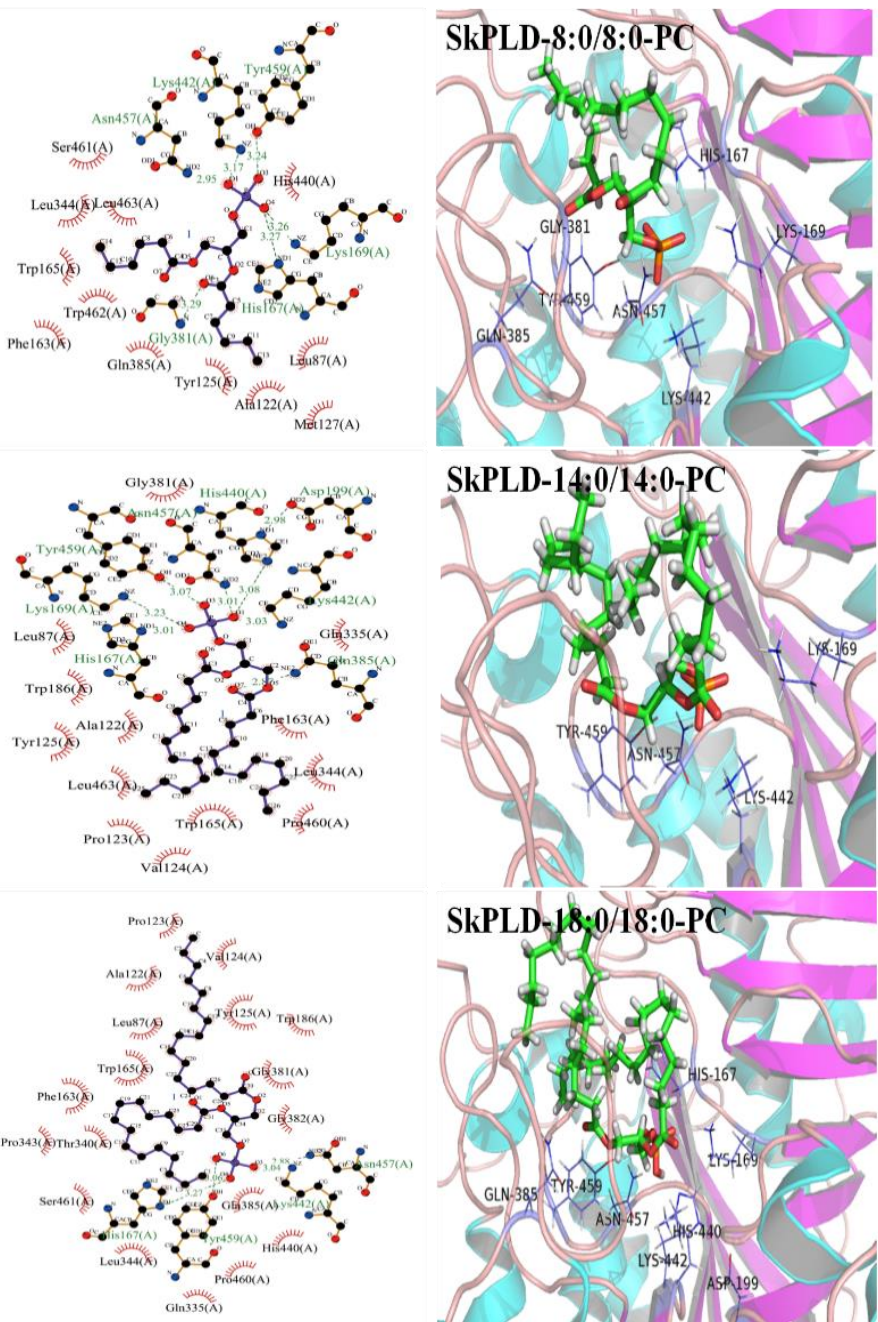
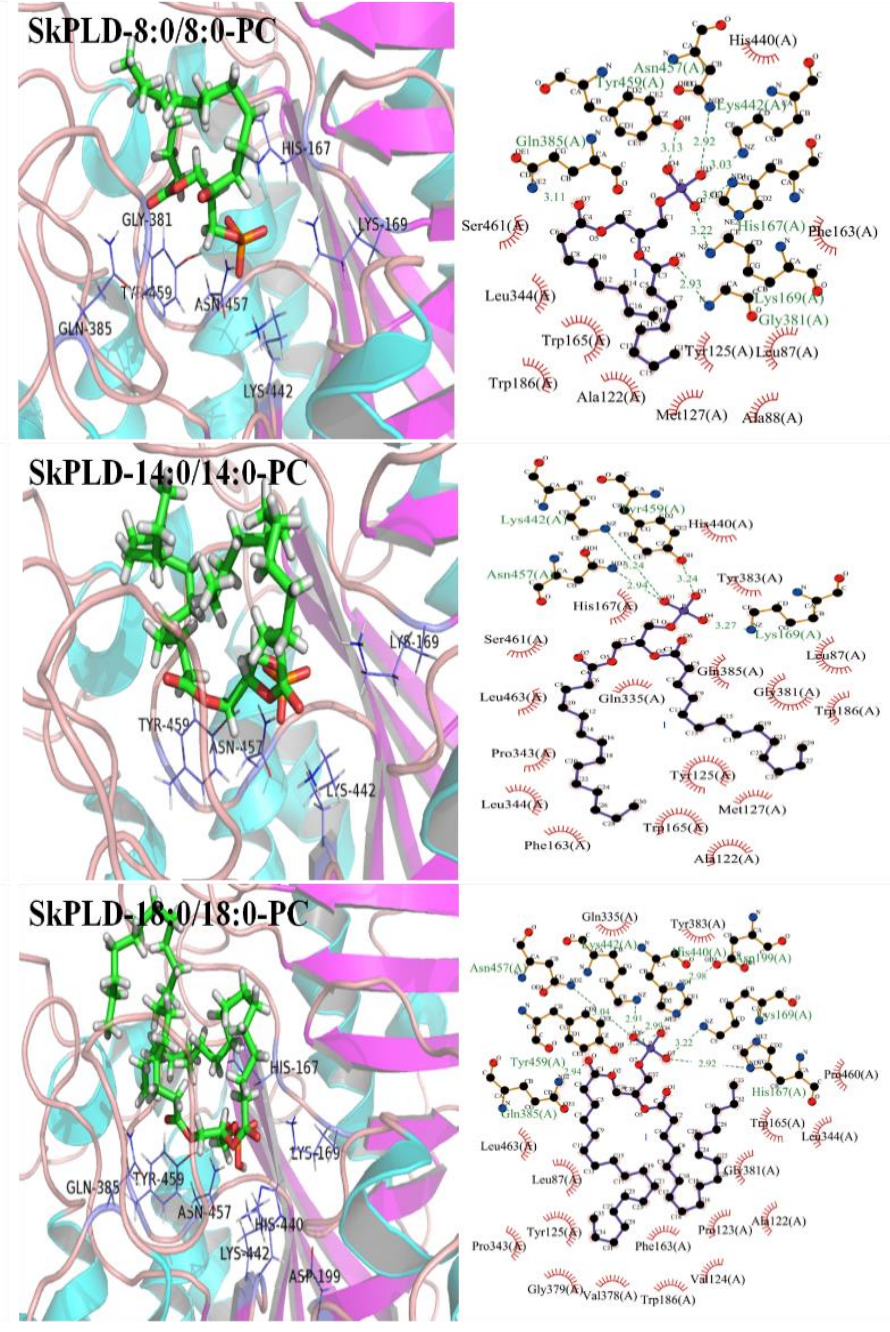

Figure 3. 3D (left) and 2D (right) structure of complexes of SkPLD and 6:0/6:0-PC, 8:0/8:0-PC, 12:0/12:0-PC, 14:0/14:0-PC, 16:0/16:0-PC and 18:0/18:0-PC. In the 2D diagram, hydrogen-bond interactions and hydrophobic contacts were shown as green and red dotted lines, respectively. 
Table 2. The estimated binding energy of SkPLD-PC complexes.

\begin{tabular}{cc}
\hline Complex & Binding Energy (kcal/mol) \\
\hline SkPLD-6:0/6:0-PC & -7.10 \\
SkPLD-8:0/8:0-PC & -7.13 \\
SkPLD-12:0/12:0-PC & -7.54 \\
SkPLD-14:0/14:0-PC & -7.29 \\
SkPLD-16:0/16:0-PC & -6.79 \\
SkPLD-18:0/18:0-PC & -7.28 \\
\hline
\end{tabular}

\subsection{Enzymatic Synthesis of PS by Recombinant SkPLD}

PLD-catalyzed synthesis of PS from soybean PC is a transphosphatidylation reaction in which PC is a donor of the phosphatidyl residue and L-serine is an acceptor. PS could be synthesized with L-serine and soybean PC as substrates under the catalysis of SkPLD. Effects of the type of organic solvent were investigated to optimize the PS yield. As shown in Figure S6, the addition of SkPLD would generate the desired product PS, as well as undesired by-product PA. As shown in Figure 4, using diethyl ether as the organic phase, with shaking $\left(200 \mathrm{rpm}\right.$ ) at $40{ }^{\circ} \mathrm{C}$ for $12 \mathrm{~h}$, the reaction showed $26.18 \%$ of yield and $1.31 \mathrm{mg} / \mathrm{mL}$ of accumulated PS concentration. The reaction in the diethyl ether afforded $1.70,4.23,1.30$ and 6.89 times PS yield as much as that of the hexane, tricholoromethane, ethyl acetate and toluene.

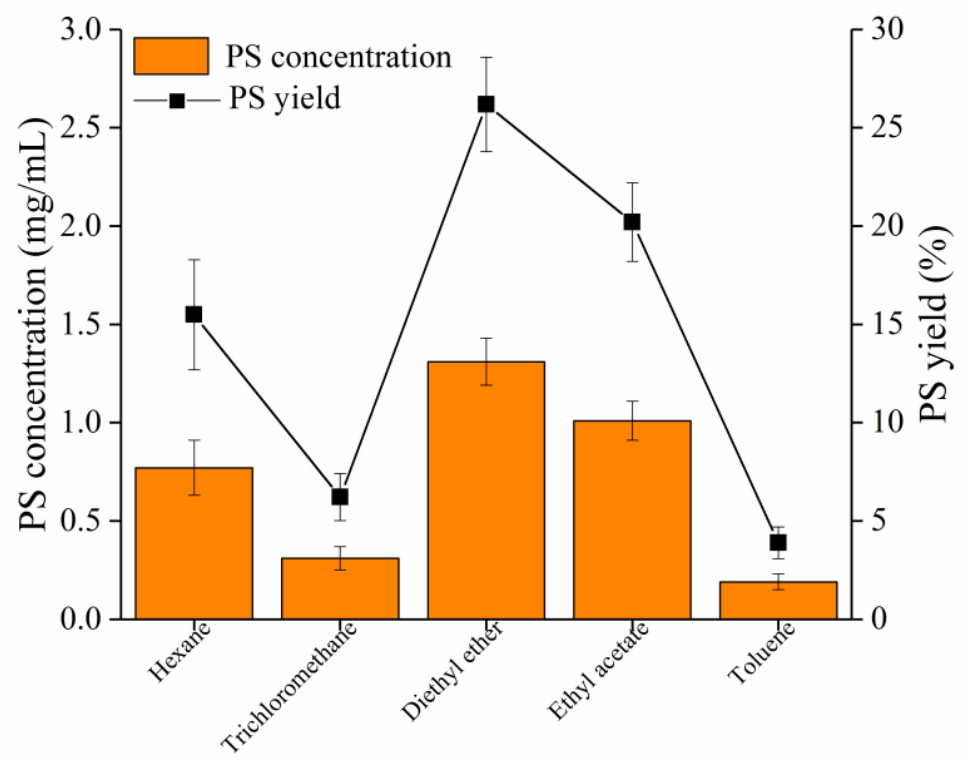

Figure 4. Application of SkPLD for PS synthesis. Effect of different organic solvents on the yield of PS. Values were means \pm standard deviation from three independent experiments.

\section{Discussion}

\subsection{Bioinformatic Analysis of SkPLD}

General findings related to the PLD catalytic reactions are a "ping-pong" type of reaction by the two HKD motifs. The histidine residue of the HKD motif in one subunit acts as the nucleophile attacking the phosphate of the phosphodiester bond, and the histidine residue in the other HKD motif acts as a general acid protonating the leaving group [16]. GG/S sequence was identified at the seven amino acids downstream of 167HXK $(\mathrm{X})_{4} \mathrm{D} 174$ and $440 \mathrm{HXK}(\mathrm{X})_{4} \mathrm{D} 447$ motif from SkPLD sequence. Two GG/S motifs patterns have also been found in other PLD sequences from Streptomyces. The PLDs from Streptomyces was known for higher catalytic activity, which was likely to benefit from the function of two GG/S motifs. Ogino et al. investigated that the G215S and G216S mutants exhibit approximately 9-16 folds higher transphosphatidylation activity than the 
wild type PLD. However, the S489G mutant show reduced activity and G488S lost the activity completely [17]. In another study, it was inferred from the crystal structure of Streptomyces sp. Strain PMF PLD that the GG/GS motif might control the active centre cleft's conformation or access [18]. These findings implied that SkPLD carrying the GG/S motifs might also recognize phospholipids more widely and affect the activity of the transphosphatidylation reaction.

\subsection{Enzymatic Characterization of SkPLD}

SkPLD showed suitable hydrolyzing activity in the $\mathrm{pH}$ range between 6.5 and 8.5, which was similar to other reported microbial PLDs from Streptomyces [19]. SkPLD showed wide $\mathrm{pH}$ stability between 7.0-10.0. However, the PLD of Streptomyces sp. PMF and Streptomyces sp. CS-57 undergo rapidly decreased activity when $\mathrm{pH}$ value was higher than $9.0[20,21]$. The maximum hydrolytic activity of SkPLD was found at $60^{\circ} \mathrm{C}$, whereas its activity decreased to $40 \%$ of the maximum under the temperature of $70{ }^{\circ} \mathrm{C}$. SkPLD kept stable after incubation at temperatures lower than $35^{\circ} \mathrm{C}$ for $4 \mathrm{~h}$, with a residual activity higher than $80 \%$. The PLD of Streptomyces sp. PMF was stable between $20^{\circ} \mathrm{C}$ and $50{ }^{\circ} \mathrm{C}$. However, while treated at $60^{\circ} \mathrm{C}$ for $3 \mathrm{~h}$, its residual activity was significantly lost [18]. The enzyme from S. halstedii $\mathrm{K} 1$ lost its activity at $45^{\circ} \mathrm{C}$. PLD from S. septatus TH-2 was stable between $15{ }^{\circ} \mathrm{C}$ and $60^{\circ} \mathrm{C}$, but decrease rapidly at $70{ }^{\circ} \mathrm{C}$ [22]. Compared to the above mentioned PLDs, SkPLD showed higher thermostabilty, especially at temperatures higher than $60^{\circ} \mathrm{C}$.

\subsection{Kinetic Parameters and Acyl Chain Specificity of SkPLD}

Knowledge of substrate specificity is essential to improve the quality of the products and to further elucidate the mechanisms of reaction. The influence of the acyl chain length in PC on the kinetic parameters of SkPLD was also investigated (Table 1). The enzymes exhibited the highest catalytic efficiency $\left(k_{\text {cat }} / K_{\mathrm{m}}\right)$ toward PCs with medium acyl chain followed by short and long acyl chain. This may be due to the increased steric hindrance of long acyl-chains in the substrate-binding pockets and differences in hydrophobic interaction between the acyl chains and substrate-binding pockets $[23,24]$. Until now, the effect of acyl chain length of PC substrates on the S. antibioticus PLD have been studied [25]. S. antibioticus PLD shows relatively high specific activity toward PCs with medium and long sn-2 acyl chain, and remarkably decreases toward PCs short sn-2 acyl chain. In summary, PCs with short and medium acyl chain were better substrates for SkPLD than those with longer chains, this characterization may be enable synergies between SkPLD and SaPLD in industrial applications that require a wider substrate range.

\subsection{Structural Feature and Molecular Basis for Chain Length Specificity}

In order to explore the molecular basis of SkPLD-mediated chain length specificity, the docking study of the substrate PC against PLD was performed, and the results are displayed in Figure 3 and Table 2. Hydrogen bond is one of the most important directional intermolecular interactions, it is responsible for the basic structures and stabilities of biomolecules [26]. Although the geometry of catalytic site is highly conserved, the binding pockets are highly variable among enzymes. The variable composition of the binding pockets generates an elastic environment for the acyl chains with various lengths [27]. The hydrogen bonds between SkPLD and PCs were initially increased with increasing length of acyl chain from 6:0/6:0-PC to 12:0/12:0-PC and then decreased with increasing length of acyl chain from 12:0/12:0-PC to 16:0/16:0-PC, which were basically consistent with its catalytic efficiency $\left(k_{\mathrm{cat}} / K_{\mathrm{m}}\right)$ and substrate affinity $\left(K_{\mathrm{m}}\right)$. This can be explained that PCs with medium chain lengths provided more binding sites to the protein chain, causing stronger hydrogen bond interactions. Generally, the hydrophobicity of the bottom of the substrate-binding pocket is important for both selectivity and recognition of hydrophobic substrates [28]. The hydrophilic regions of substrate-binding pocket of SkPLD were mainly located in the amino acid sequences of 381-385. Compared with PCs with short and 
medium acyl chain lengths, the hydrophobic acyl chain of 16:0/16:0-PC was in contacted with the hydrophilic regions (Gly381, Gly382 and Gln385) of substrate-binding pocket of SkPLD. These contacts may weaken hydrophobic interactions and which are detrimental to hydrogen bond formation and complex stability [29]. SkPLD and 18:0/18:0-PC complex obtained binding energy of $-7.28 \mathrm{kcal} / \mathrm{mol}$ and relatively high hydrogen-bond interaction, which was consistent with its substrate affinity $\left(K_{\mathrm{m}}\right)$. However, SkPLD exhibited low catalytic efficiency for 18:0/18:0-PC. This can be explained that the increased steric hindrance of long acyl-chains in the substrate-binding pockets and it was more difficult for PC molecule to enter and escape the binding pocket. These results indicated that PCs with shorter chain length provided less binding sites to the protein chain, causing weaker interactions or binding, while the PCs with long chain length increased steric hindrance in the substrate-binding pocket.

\subsection{Enzymatic Synthesis of PS by Recombinant SkPLD}

Since both the substrate PC and the product PS are water-insoluble while the substrate L-serine is water-soluble, this enzyme-catalyzed transphosphatidylation generally was carried out in a biphasic system consisting of a water-immiscible organic solvent phase [30]. Plenty of organic solvents have been well investigated for the Streptomyce PLD-mediated transphosphatidylation for PS preparation, including diethyl ether, ethyl acetate, chloroform and dichloromethane. S. racemochromogenes PLD used dichloromethane as preferred organic solvent phase [31]. Streptomyces sp. SC734 PLD and Streptomyces chromofuscus AS 4.331 used chloroform as preferred organic solvent phase [32,33]. Streptomyces sp. CA-1 and Streptomyces sp. used ethyl acetate as preferred organic solvent phase [34,35]. Notably, most of Streptomyces PLD have achieved more than 50\% of PS using diethyl ether as organic solvent phase, such as Streptomyces sp. YU100, Streptomyces prunicolor, Streptomyces sp. LD0501 [36,37]. Although diethyl ether system have achieved decent yields of PS, the enzymatic reaction is greatly affected by plenty of factors, such as the choice of organic solvent, the volume ratio of biphasic system, the proportion of substrate concentration, $\mathrm{pH}$, temperature, which make it much complicated [38]. Moreover, diethyl ether has certain toxicities which lead to enzyme denaturation [39]. Therefore, further optimization of Streptomyces PLD-catalyzed transphosphatidylation condition, including culture time, the volume ratio of biphasic system, $\mathrm{pH}$ and temperature are necessary for industrial applications in PS production.

\section{Materials and Methods}

\subsection{Chemicals, Strains, and Materials}

PC (purity 95\%, from soybean), 1, 2-dihexanoyl-sn-glycero-3-phosphocholine (6:0/6:0-PC) (purity $>99 \%$ ), 1, 2-dioctanoyl-sn-glycero-3-phosphocholine (8:0/8:0-PC) (purity > 99\%), 1 , 2-dilauroyl-sn-glycero-3-phosphocholine (12:0/12:0-PC) (purity > 99\%), 1, 2-dimyristoylsn-glycero-3-phosphocholine (14:0/14:0-PC) (purity > 99\%), 1, 2-di-palmitoyl-sn-glycero-3phosphocholine (16:0/16:0-PC) (purity > 99\%) and 1, 2-distearoyl-sn-glycerol-3-phosphocholine (18:0/18:0-PC) (purity > 99\%) were purchased from Avanti Polar Lipids, Inc. (Alabaster, AL, USA). PS standard (purity $>97 \%$ ) was purchased from Sigma-Aldrich (St. Louis, MO, USA). Choline oxidase was prepared by the previously reported method [40]. Horseradish peroxidase was purchased from Sangon Biotech Co., Ltd. (Shanghai, China). Hypersil GOLD Silica column (Dim. $4.6 \times 250 \mathrm{~mm}$, particle size $5.0 \mu \mathrm{m}$ ) was obtained from Thermo Fisher Scientific (Waltham, MA, USA). Escherichia coli SHuffle T7 Express competent cell were purchased from New England BioLabs (Beijing, China). The plasmid pET28a vector was purchased from Invitrogen (Carlsbad, CA, USA). $\mathrm{Ni}^{2+}$-nitrilotriacetate $\left(\mathrm{Ni}^{2+}-\mathrm{NTA}\right)$ affinity column, desalting column, Q-Sepharose column and Hiload 16/60 Superdex 200 pg gel filtration column were obtained from GE Healthcare Life Sciences (Pittsburgh, PA, USA). All other chemicals used in the present study were analytical grade. 


\subsection{Bioinformatic and Homology Modeling}

The SkPLD protein from marine Streptomyces klenkii that expressed in this study was deposited in the NCBI-Protein databases under the accession number of RKN69773.1. The signal peptide of SkPLD was predicted using online tools SignaIP v. 5.0 web server (http:/ / www.cbs.dtu.dk/services/SignalP/, accessed on 20 March 2020) [41]. The mature peptide (34 to 539aa, without signal peptide) of SkPLD protein was codon optimization with the E. coli code usage (Figure S7). Sequence comparison of SkPLD to other known PLDs was performed by Multalin (http:/ / multalin.toulouse.inra.fr/multalin/, accessed on 20 March 2020) [42]. Homology modeling of SkPLD was performed using the SWISSMODEL (https: / / swissmodel.expasy.org, accessed on 20 March 2020) online system [43]. The protein three-dimensional structure of PDB entry 2ZE9 with the sequence identity of $75.56 \%$ to SkPLD was used as a template [44]. The validation and quality estimation of predicted SkPLD model was evaluated by QMEAN [45]. The ligand and protein files were prepared using AutoDock tools [46]. AutoDock tools was used for protein-ligand docking and the resulting interactions of between receptor and ligand were visualized PyMOL (version 2.5) [47] and LigPlus (version 2.2) [48].

\subsection{Recombinant Expression of SkPLD in Escherichia coli}

The gene encoding the mature protein of SkPLD without the signal peptide was artificially synthesized by Sangon Biotech Co., Ltd. (Shanghai, China) and inserted into the pET-28a expression vector. Plasmids were confirmed by sequencing. The constructed vector was further transformed into Shuffle T7 express competent E. coli. A single colony of E. coli SHuffle T7 cells harbouring the plasmid pET-28a-SkPLD was grown at $37^{\circ} \mathrm{C}$ for $12 \mathrm{~h}$ in $5 \mathrm{~mL}$ of Luria-Bertani medium containing $50 \mu \mathrm{g} / \mathrm{mL}$ kanamycin. An aliquot $(5 \mathrm{~mL})$ of this culture was inoculated into $500 \mathrm{~mL}$ of the same medium and grown at $37^{\circ} \mathrm{C}$ with shaking $\left(220 \mathrm{rpm}\right.$ ). When the $\mathrm{OD}_{600}$ value reached 0.6 (Metash UV-6000PC, Shanghai, China), enzyme expression was induced by adding isopropyl- $\beta$-D-thiogalactopyranoside (IPTG) with a final concentration of $1 \mathrm{mM}$. After cultivation was continued for another $12 \mathrm{~h}$ at $16^{\circ} \mathrm{C}$, cells were harvested by centrifugation (Hermle Z326K, Wehingen, Germany) at $8000 \times \mathrm{g}$ for $10 \mathrm{~min}$ at $4{ }^{\circ} \mathrm{C}$ and stored at $-20^{\circ} \mathrm{C}$ for further use.

\subsection{Purification of Recombinant SkPLD}

The SkPLD was purified by metal-chelating chromatography using a $\mathrm{Ni}^{2+}$-nitrilotriacetate affinity column. Buffer A ( $50 \mathrm{mM}$ Tris- $\mathrm{HCl}, 20 \mathrm{mM}$ imidazole, $\mathrm{pH}$ 8.0) containing crude enzyme was loaded into a $\mathrm{Ni}^{2+}$-nitrilotriacetate affinity column and washed with buffer B (50 mM Tris- $\mathrm{HCl}, 250 \mathrm{mM}$ imidazole, $\mathrm{pH} 8.0)$. The eluant was buffer exchanged to $20 \mathrm{mM}$ Tris- $\mathrm{HCl}(\mathrm{pH} 8.0)$ through a desalting column. And then applied to a Q-column pre-equilibrated with $20 \mathrm{mM}$ Tris- $\mathrm{HCl}, \mathrm{pH}$ 8.0. The elution was performed at the flow rate of $1 \mathrm{~mL} / \mathrm{min}$ with a gradient of $\mathrm{NaCl}$ from 0 to $1 \mathrm{M}$, in $20 \mathrm{mM}$ Tris- $\mathrm{HCl}, \mathrm{pH}$ 8.0. Then we knowed that buffer containing $700 \mathrm{mM} \mathrm{NaCl}$ can elude the target protein. Therefore, we eluted the non-target protein with buffer containing $500 \mathrm{mM} \mathrm{NaCl}$, and then eluted the target protein with buffer containing $700 \mathrm{mM} \mathrm{NaCl}$ to obtain a relatively pure target protein. The eluted sample from Q-column chromatography with elution buffer containing $700 \mathrm{mM}$ $\mathrm{NaCl}$ was buffer exchanged to $20 \mathrm{mM}$ Tris- $\mathrm{HCl}(\mathrm{pH} 8.0)$ through a desalting column and then applied to a Hiload 16/60 Superdex 200 pg. Finally, target protein fractions were further pooled after size-exclusion chromatography. The absorbance of the elution was monitored at $280 \mathrm{~nm}$.

\subsection{Enzyme Activity Assay}

The PLD activity was assayed using the method described by Shimbo et al. with minor modifications [49]. For substrate preparations, $1 \mathrm{mmol}$ of soybean PC or corresponding amounts of other phospholipids were dissolved in $5 \mathrm{~mL}$ of chloroform. After evaporation to dryness, SDS and Triton X-100 were added to yield a solution containing $10 \mathrm{mM} \mathrm{PC}$, $12.5 \mathrm{mM}$ SDS, and $40 \mathrm{mM}$ Triton X-100. The solution was vortexed and sonicated for 
$10 \mathrm{~min}$ using a probe sonicator $(800 \mathrm{~W} / \mathrm{s})$. The reaction mixture $(100 \mu \mathrm{L})$ consisted of $4 \mathrm{mM}$ soybean PC, $50 \mathrm{mM}$ Tris- $\mathrm{HCl}$ (pH 8.0), $20 \mathrm{mM} \mathrm{CaCl}_{2}$ and $10 \mu \mathrm{L}$ of an enzyme sample. After incubation at $40{ }^{\circ} \mathrm{C}$ for $10 \mathrm{~min}$ with shaking, reaction was terminated by adding $25 \mu \mathrm{L}$ Tris-HCl (50 mM, pH 8.0) solution that contained $50 \mathrm{mM}$ EDTA. The PLD was further denatured by heating at $100{ }^{\circ} \mathrm{C}$ for $5 \mathrm{~min}$. After cooling the reaction mixture to room temperature for $5 \mathrm{~min}, 25 \mu \mathrm{L}$ of Tris- $\mathrm{HCl}(50 \mathrm{mM}, \mathrm{pH} 8.0)$ containing $42 \mathrm{mM}$ phenol, $50 \mathrm{mM}$ 4-aminoantipyrine, $0.5 \mathrm{U}$ of horseradish peroxidase and $0.25 \mathrm{U}$ of choline oxidase was added. After incubation at $37^{\circ} \mathrm{C}$ for $60 \mathrm{~min}$, the absorbance of the reaction mixture was measured at $500 \mathrm{~nm}$ (Tecan Infinite 200 PRO, Männedorf, Switzerland). As a blank test, $10 \mu \mathrm{L}$ of distilled water was used instead of the enzyme solution, and the reaction mixture was treated in the same way. The calibration curve was obtained using a standard solution of choline chloride instead of the enzyme solution. One unit (U) of hydrolytic activity of PLD was defined as the amount of enzyme producing $1 \mu \mathrm{mol}$ of choline per minute under these assay conditions. All assays were performed in triplicate.

\subsection{Enzymatic Characterization of SkPLD}

Optimum $\mathrm{pH}$ for the purified SkPLD activity was determined at $40{ }^{\circ} \mathrm{C}$ using soybean PC as substrate. $\mathrm{pH}$ buffer solutions ranged from 5.0 to 10.0 ( $\mathrm{pH}$ 5.0, $0.1 \mathrm{M}$ sodium citratecitric acid; $\mathrm{pH}$ 6.0-7.0, 0.1 M phosphate buffer; $\mathrm{pH}$ 8.0, 0.1 $\mathrm{M}$ Tris- $\mathrm{HCl}$ and $\mathrm{pH}$ 9.0-10.0, $0.1 \mathrm{M}$ Gly-NaOH). pH stability of SkPLD was determined by pre-incubating enzymes in different $\mathrm{pH}$ buffers for $2 \mathrm{~h}$ at $25^{\circ} \mathrm{C}$, and then the residual activity was determined at $40{ }^{\circ} \mathrm{C}$ and optimum $\mathrm{pH}$. The optimum temperature of the SkPLD activity was determined at optimum $\mathrm{pH}$. The temperature range was set from $30-80^{\circ} \mathrm{C}$. The thermostability of SkPLD was determined by pre-incubating the enzyme at different temperatures and $\mathrm{pH} 8.0$, and then samples were taken every $60 \mathrm{~min}$ for measurement of residual activity under the above assay optimum conditions. The temperature was set as $25,35,45,55,65$ and $75{ }^{\circ} \mathrm{C}$, respectively. Various metal ions $\left(\mathrm{Mg}^{2+}, \mathrm{Co}^{2+}, \mathrm{Ca}^{2+}, \mathrm{Na}^{+}, \mathrm{Mn}^{2+}, \mathrm{Al}^{3+}, \mathrm{K}^{+}, \mathrm{Cu}^{2+}, \mathrm{Li}^{+}, \mathrm{Zn}^{2+}\right.$, $\mathrm{Fe}^{2+}$ and $\mathrm{Fe}^{3+}$ ) and EDTA at final concentrations of $10 \mathrm{mM}$ were added to the enzyme at $\mathrm{pH} 8.0$, and then the PLD activity was assayed after pre-incubation at $25^{\circ} \mathrm{C}$ for $2 \mathrm{~h}$. The enzyme activity in the absence of metal ions and EDTA were defined as $100 \%$.

The kinetic parameters of SkPLD to soybean PC and PC with different defined fatty acids (6:0/6:0, 8:0/8:0, 12:0/12:0, 14:0/14:0, 16:0/16:0, 18:0/18:0) as the substrates were determined by using enzyme-linked colorimetry with different substrate concentrations $(0-4 \mathrm{mM})$. The affinity constant $\left(K_{\mathrm{m}}\right)$, maximal velocity $\left(V_{\max }\right)$, and turnover number $\left(k_{\text {cat }}\right)$ values of the reaction were obtained using the Michaelis-Menten equation. The independently replicated determination was performed three times for each substrate. The error bars represent three independent assays of kinetic parameters.

\subsection{Application of SkPLD for the PS Synthesis}

Reactions were performed in a biphasic reaction system. The initial conversion conditions were as follows: a reaction mixture consisting of $20 \mathrm{mg} / \mathrm{mL}$ L-serine in $500 \mu \mathrm{L}$ enzyme solution (100 $\mu \mathrm{g} / \mathrm{mL}$ PLD in $100 \mathrm{mM}$ Tris-HCl buffer, $\mathrm{pH}$ 8), and soybean PC $\left(5 \mathrm{mg} / \mathrm{mL}\right.$ ) dissolved in $500 \mu \mathrm{L}$ of organic solvent was incubated at $40{ }^{\circ} \mathrm{C}$ for $12 \mathrm{~h}$ with shaking. To obtain the optimum PLD-catalyzed transphosphatidylation conditions, the effects of various organic solvent (hexane, ethyl acetate, chloroform, ether and toluene) on the yield of PS were investigated. For quantification, PS was detected by Waters 1525 HPLC (Milford, MA, USA) equipped with ELSD. Reaction mixture was eluted with a column using solvent A (methanol/water/acetic acid/triethylamine, 425:75:2.5:0.25, by vol.) and solvent B (n-hexane/isopropanol/solvent A, 160:384:256, by vol.). The elution profile was as follows: $0 \mathrm{~min}, \mathrm{~A}=100 \% ; 9 \mathrm{~min}$, B increased to $40 \% ; 13 \mathrm{~min}$, B increased to $60 \% ; 17 \mathrm{~min}$, $\mathrm{B}=100 \% ; 22 \mathrm{~min}$ decreased to $0 \%$. The yield of PS (\%) was defined as the percentage of PS obtained compared with the initial PC concentration. 


\section{Conclusions}

In summary, a new PLD from marine Streptomyces klenkii was biochemical characterized. PCs with short and medium acyl chain were better substrates for SkPLD than those with longer chains. The different catalytic efficiency of SkPLD toward PCs with various chain lengths was related to the increased steric hindrance of long acyl-chains in the substrate-binding pockets and differences in hydrogen-bond interactions between the acyl chains and substrate-binding pockets. Present investigations not only further enriched the enzyme library but also provide guidance for the further mining of PLDs with special phospholipids recognition properties.

Supplementary Materials: The following are available online at https://www.mdpi.com/article/10 .3390/ijms221910580/s1.

Author Contributions: R.H.: Investigation, Formal analysis, Writing-original draft. R.C.: Formal analysis. D.L.: Supervision, Formal analysis. F.W.: Supervision, Formal analysis. Y.W.: WritingReview \& Editing, Supervision, Funding acquisition. All authors have read and agreed to the published version of the manuscript.

Funding: This work was supported by the National Key R \& D Program of China (2018YFC0311104), National Science Fund for Distinguished Young Scholars (31725022), Key Program of Natural Science Foundation of China (31930084), Guangdong marine economy promotion projects (MEPP) Fund (no. GDOE[2019]A20) and National Natural Science Foundation of China (31972014, 32001639).

Institutional Review Board Statement: Not applicable.

Informed Consent Statement: Not applicable.

Data Availability Statement: The data presented in this study are available on request from the corresponding author.

Conflicts of Interest: The authors declare no conflict of interest.

\section{References}

1. Kim, H.-Y.; Huang, B.X.; Spector, A.A. Phosphatidylserine in the brain: Metabolism and function. Prog. Lipid Res. 2014, 56, 1-18. [CrossRef]

2. Haq, M.; Suraiya, S.; Ahmed, S.; Chun, B.S. Phospholipids from Marine Source: Extractions and Forthcoming Industrial Ap-plications. J. Funct. Foods 2021, 80, 104448. [CrossRef]

3. Ric, D.; Élodie, B.; Habib, H.; Daniel, B.; Philippe, C.; Line, C.; Nicolas, B.; Sophie, C.; Bernard, D.; Christian, S. Lipid Selectivity, Orientation, and Extent of Membrane Binding of Nonacylated RP2. Biochemistry 2015, 54, 2560-2570.

4. Qin, W.; Wu, C.J.; Song, W.; Chen, X.L.; Liu, J.; Luo, Q.L.; Liu, L.M. A Novel High-Yield Process of Phospholipase D-Mediated Phosphatidylserine Production with Cyclopentyl Methyl Ethe. Process. Biochem. 2018, 66, 146-149. [CrossRef]

5. Li, B.; Duan, D.; Wang, J.; Li, H.; Zhang, X.; Zhao, B. Improving phospholipase D activity and selectivity by bio-imprintingimmobilization to produce phosphatidylglycerol. J. Biotechnol. 2018, 281, 67-73. [CrossRef] [PubMed]

6. Deng, Y.; Li, B.; Dong, W.; Yang, W.; Zhang, X.; Zhao, B. Synthesis of Phosphatidylethanolamine by Enzymatic Catalysis and Its Substrate Inhibition Kinetics. Chem. Ind. Eng. Prog. 2017, 36, 2601-2606.

7. Shirouchi, B.; Nagao, K.; Inoue, N.; Furuya, K.; Koga, S.; Matsumoto, H.; Yanagita, T. Dietary Phosphatidylinositol Prevents the Development of Nonalcoholic Fatty Liver Disease in Zucker (fa/fa) Rats. J. Agric. Food Chem. 2008, 56, 2375-2379. [CrossRef] [PubMed]

8. Cao, K.; Liu, Y.; Tian, Y.; Zhang, Q.; Cong, P.; Li, H.; Xu, J.; Li, Z.; Wang, J.; Mao, X. Reaction Specificity of Phospholipase D Prepared from Acinetobacter radioresistens a2 in Transphosphatidylation. Lipids 2018, 53, 517-526. [CrossRef] [PubMed]

9. Laouini, A.; Jaafar-Maalej, C.; Limayem-Blouza, I.; Sfar, S.; Charcosset, C.; Fessi, H. Preparation, characterization and applications of liposomes: State of the art. J. Colloid Sci. Biotechnol. 2012, 1, 147-168. [CrossRef]

10. Ogino, C.; Kuroda, S.; Tokuyama, S.; Kondo, A.; Shimizu, N.; Tanizawa, K.; Fukuda, H. Phospholipase D from Streptoverti-cillium cinnamoneum: Protein Engineering and Application for Phospholipid Production. J. Mol. Catal. B Enzym. 2003, 23, 107-115. [CrossRef]

11. Ulbrich-Hofmann, R.; Lerchner, A.; Oblozinsky, M.; Bezakova, L. Phospholipase D and its application in biocatalysis. Biotechnol. Lett. 2005, 27, 535-544. [CrossRef]

12. Zerrifi, S.E.A.; Redouane, E.M.; Mugani, R.; Ribeiro, I.; Carvalho, M.F.; Campos, A.; Barakate, M.; Vasconcelos, V.; Oudra, B.; El Khalloufi, F. Moroccan actinobacteria with promising activity against toxic cyanobacteria Microcystis aeruginosa. Environ. Sci. Pollut. Res. 2020, 28, 235-245. [CrossRef] 
13. Yuan, D.J.; Lan, D.M.; Xin, R.P.; Yang, B.; Wang, Y.H. Screening and Characterization of a Thermostable Lipase from Marine Streptomyces sp. strain W007. Biotechnol. Appl. Biochem. 2016, 63, 41-50. [CrossRef] [PubMed]

14. Zhou, W.-B.; Gong, J.-S.; Hou, H.-J.; Li, H.; Lu, Z.-M.; Xu, H.-Y.; Xu, Z.-H.; Shi, J.-S. Mining of a phospholipase D and its application in enzymatic preparation of phosphatidylserine. Bioengineered 2017, 9, 80-89. [CrossRef] [PubMed]

15. Waite, M. The PLD superfamily: Insights into catalysis. Biochim. Biophys. Acta (BBA)-Mol. Cell Biol. Lipids 1999, $1439,187-197$. [CrossRef]

16. Wang, J.; Li, B.; Zhang, X.; Hu, Q.; Yu, W.; Wang, H.; Duan, D.; Li, J.; Zhao, B. Docking and molecular dynamics studies on the mechanism of phospholipase D-mediated transphosphatidylation to construct the reaction kinetic model: Application in phosphatidylserine production. J. Taiwan Inst. Chem. Eng. 2018, 96, 82-92. [CrossRef]

17. Ogino, C.; Daido, H.; Ohmura, Y.; Takada, N. Remarkable enhancement in PLD activity from Streptoverticillium cinnamoneum by substituting serine residue into the GG/GS motif. Biochim. Biophys. Acta (BBA)-Proteins Proteom. 2007, 1774, 671-678. [CrossRef]

18. Leiros, I.; Secundo, F.; Zambonelli, C.; Servi, S.; Hough, E. The first crystal structure of a phospholipase D. Structure 2000, 8, 655-667. [CrossRef]

19. Zhang, Z.; Chen, M.; Xu, W.; Zhang, W.; Zhang, T.; Guang, C.; Mu, W. Microbial phospholipase D: Identification, modification and application. Trends Food Sci. Technol. 2020, 96, 145-156. [CrossRef]

20. Zambonellia, C.; Morandib, P.; Vanonib, M.A.; Tedeschic, G.; Servia, S.; Curtib, B. Cloning and expression in Escherichia coli of the gene encoding Streptomyces PMF PLD, a phospholipase D with high transphosphatidylation activity. Enzym. Microb. Technol. 2003, 33, 676-688. [CrossRef]

21. Simkhada, J.R.; Cho, S.S.; Lee, H.J.; Yoo, J.C. Purification and biochemical properties of phospholipase d (PLD57) produced by Streptomyces sp. CS-57. Arch. Pharmacal Res. 2007, 30, 1302-1308. [CrossRef]

22. Hatanaka, T.; Negishi, T.; Kubota-Akizawa, M.; Hagishita, T. Study on thermostability of phospholipase D from Streptomyces sp. Biochim. Biophys. Acta (BBA)-Proteins Proteom. 2002, 1598, 156-164. [CrossRef]

23. Yasmeen Yousif Ahmed, E.; Kazusa, M.; Katsuhiko, S.; Jiro, A. Effect of Active Site Pocket Structure Modification of DStereospecific Amidohydrolase on the Recognition of Stereospecific and Hydrophobic Substrates. Mol. Biotechnol. 2018, 60, 690-697.

24. Gaskin, D.J.H.; Romojaro, A.; Turner, N.A.; Jenkins, J.; Vulfson, E.N. Alteration of lipase chain length specificity in the hydrolysis of esters by random mutagenesis. Biotechnol. Bioeng. 2001, 73, 433-441. [CrossRef] [PubMed]

25. Jasmina, D.; Hideo, N.; Yugo, I. Acyl chain that matters: Introducing sn-2 acyl chain preference to a phospholipase D by protein engineering. Protein Eng. Des. Sel. 2019, 32, 1-11.

26. Lee, J.S. Binding energies of hydrogen-bonded complexes from extrapolation with localized basis sets. J. Chem. Phys. 2007, 127, 08B616. [CrossRef]

27. Pleiss, J.; Fischer, M.; Schmid, R.D. Anatomy of lipase binding sites: The scissile fatty acid binding site. Chem. Phys. Lipids 1998, 93, 67-80. [CrossRef]

28. Traul, K.; Driedger, A.; Ingle, D.; Nakhasi, D. Review of the toxicologic properties of medium-chain triglycerides. Food Chem. Toxicol. 2000, 38, 79-98. [CrossRef]

29. Qin, X.; Zhong, J.; Wang, Y. A mutant T1 lipase homology modeling, and its molecular docking and molecular dynamics simulation with fatty acids. J. Biotechnol. 2021, 337, 24-34. [CrossRef]

30. Chen, S.; Xu, L.; Li, Y.; Hao, N.; Yan, M. Bioconversion of Phosphatidylserine by Phospholipase D from Streptomyces racemochromogenes in a Microaqueous Water-Immiscible Organic Solvent. J. Agric. Chem. Soc. Jpn. 2013, 77, 1939-1941.

31. Qian, J.; Yang, P.; Wang, X. Preparation of phospholipase D and catalytic synthesis of phosphatidylserine from phosphatidylcholine. China Oil Fat. 2017, 42, 62-71.

32. Saovanee, C.; Uwe, T.B.; Apichat, U.; Aran, H.K. Efficient phosphatidylserine synthesis by a phospholipase D from Strepto-myces sp. SC734 isolated from soil-contaminated palm oil. Eur. Lipid Sci. Technol. 2016, 118, 803-813.

33. Li, B.; Lu, F.P.; Tian, L.; Li, Y.; Du, L. Cloning and expression of phospholipase D gene pld from Streptomyces chromofuscus. Ann. Microbiol. 2008, 58, 227-231. [CrossRef]

34. Han, H.X.; Cao, D.; Shi, S.J.; Wang, Q. Optimization of fermentation conditions of phospholipase D from Streptomyces sp. CA-1. Sci. Technol. Food Ind. 2014, 35, 176-180.

35. Hosokawa, M.; Shimatani, T.; Kanada, T.; Inoue, Y.; Takahashi, K. Conversion to docosahexaenoic acid-containing phosphatidylserine from squid skin lecithin by phospholipase D-mediated transphosphatidylation. J. Agric. Food Chem. 2000, 48, 4550-4554. [CrossRef]

36. Lee, J.S.; Bat-Ochir, M.; Demirev, A.V.; Nam, D.H. Molecular cloning of the phospholipase D gene from Streptomyces sp. YU100 and its expression in Escherichia coli. J. Microb. 2009, 47, 116-122. [CrossRef] [PubMed]

37. Yang, S.-L.; Duan, Z.-Q. Insight into enzymatic synthesis of phosphatidylserine in deep eutectic solvents. Catal. Commun. 2016, 82, 16-19. [CrossRef]

38. Li, B.; Wang, J.; Zhang, X.; Zhao, B.; Niu, L. Aqueous-Solid System for Highly Efficient and Environmentally Friendly Transphosphatidylation Catalyzed by Phospholipase D To Produce Phosphatidylserine. J. Agric. Food Chem. 2016, 64, 7555-7560. [CrossRef]

39. Zhang, X.; Li, B.; Wang, J.; Li, H.; Zhao, B. High-Yield and Sustainable Production of Phosphatidylserine in Purely Aqueous Solutions via Adsorption of Phosphatidylcholine on Triton-X-100-Modified Silica. J. Agric. Food Chem. 2017, 65, 10767-10774. [CrossRef] 
40. Ribitsch, D.; Karl, W.; Wehrschütz-Sigl, E.; Tutz, S.; Remler, P.; Weber, H.; Gruber, K.; Stehr, R.; Bessler, C.; Hoven, N.; et al. Heterologous Expression and Characterization of Choline Oxidase from the Soil Bacterium Arthrobacter nicotianae. Appl. Microbiol. Biotechnol. 2008, 81, 875-886. [CrossRef]

41. Armenteros, J.J.A.; Tsirigos, K.; Sønderby, C.K.; Petersen, T.N.; Winther, O.; Brunak, S.; Von Heijne, G.; Nielsen, H. SignalP 5.0 improves signal peptide predictions using deep neural networks. Nat. Biotechnol. 2019, 37, 420-423. [CrossRef]

42. Florence, C. Multiple Sequence Alignment with Hierarchical Clustering. Nucleic Acids Res. 1998, 16, 10881-10890.

43. Andrew, W.; Martino, B.; Stefan, B.; Gabriel, S.; Gerardo, T.; Rafal, G.; Florian, T.H.; de Tjaart, A.P.B.; Christine, R.; Lorenza, B.; et al. SWISS-MODEL: Homology modelling of protein structures and complexes. Nucleic Acids Res. 2018, 46, W296-W303.

44. Suzuki, A.; Kakuno, K.; Satio, R.; Iwasaki, Y.; Yamane, T. Crystal Structure of Phospholipase D from Streptomyces antibioticus. Acta Crystallogr. Sect. A Found. Crystallogr. 2000, 56, s242. [CrossRef]

45. Benkert, P.; Biasini, M.; Schwede, T. Toward the estimation of the absolute quality of individual protein structure models. Bioinformatics 2010, 27, 343-350. [CrossRef] [PubMed]

46. Sussman, J.L.; Abola, E.E.; Lin, D.; Jiang, J.; Manning, N.O.; Prilusky, J. The Protein Data Bank. Genetica 1999, 106, 149-158. [CrossRef] [PubMed]

47. DeLano, W.L. The PyMOL Molecular Graphics System; Delano Scientific: San Carlos, CA, USA, 2002.

48. Laskowski, R.A.; Swindells, M.B. LigPlot+: Multiple Ligand-Protein Interaction Diagrams for Drug Discovery. J. Chem. Inf. Model. 2011, 51, 2778-2786. [CrossRef] [PubMed]

49. Shimbo, K.; Yano, H.; Miyamoto, Y. Purification and Properties of Phospholipase D from Streptomyces lydicus. Agric. Biol. Chem. 1990, 54, 1189-1193. [CrossRef] 